\title{
Clinical and Technical Characteristics of Coronary Angiography and Percutaneous Coronary Interventions Performed before and after Transcatheter Aortic Valve Replacement with a Balloon-Expandable Valve
}

\author{
Alfredo Nunes Ferreira-Neto (D), Rishi Puri, Lluis Asmarats, Victoria Vilalta, \\ Leonardo Guimaraes, Robert Delarochellière, Jean-Michel Paradis, Siamak Mohammadi, \\ Eric Dumont, and Josep Rodés-Cabau (iD)
}

Quebec Heart \& Lung Institute, Laval University, Quebec City, Quebec, Canada

Correspondence should be addressed to Josep Rodés-Cabau; josep.rodes@criucpq.ulaval.ca

Received 29 April 2019; Accepted 25 July 2019; Published 8 August 2019

Academic Editor: Andrea Rubboli

Copyright (C) 2019 Alfredo Nunes Ferreira-Neto et al. This is an open access article distributed under the Creative Commons Attribution License, which permits unrestricted use, distribution, and reproduction in any medium, provided the original work is properly cited.

\begin{abstract}
Objectives. To report on the feasibility and technical differences between coronary procedures performed before and after TAVR with the balloon-expandable Edwards-SAPIEN or the SAPIEN XT valves. Background. Coronary artery disease (CAD) and aortic stenosis often coexist. Transcatheter aortic valve replacement (TAVR) is emerging as a treatment for younger and lower surgical risk patients who might not present with clinically evident CAD before TAVR. The demand for performing post-TAVR coronary angiograms (CAs) and percutaneous coronary interventions (PCIs) will thus increase, posing new technical challenges. Methods. Over 1000 TAVRs were performed at the Quebec Heart and Lung Institute, of which 616 with the abovementioned valves. Of these, 28 patients had an analyzable pre- and post-TAVR CAs and 13 patients had pre- and post-TAVR PCIs performed. Procedural characteristics were gathered from all coronary procedures and subsequently compared amongst the same type of procedure performed at these two distinct time periods. Results. Neither CAs-nor PCIs-performed after valve implantation revealed significant differences regarding arterial access site, catheter diameter, number of diagnostic or guiding catheters used, procedural duration, fluoroscopy time, or achievement of selective coronary injection. Lesion location and classification, as well as the preference of using a drug-eluting stent, remained unchanged. During post-TAVR CA, the amount of contrast delivered and the radiation dose area product were significantly lower compared with pre-TAVR CA values. Conclusions. Performance of CA and PCI after TAVR with a balloon-expandable valve appears unaffected by its presence.
\end{abstract}

\section{Introduction}

There is considerable overlap in both the pathophysiology and presence of atherosclerotic coronary artery disease (CAD) and calcific aortic stenosis (AS), with concomitant significant CAD observed in up to $50 \%$ of individuals presenting with severe AS [1]. Given the establishment of transcatheter aortic valve replacement (TAVR) and its inevitable expansion towards the treatment of younger and lower surgical risk AS patients [2], the likelihood of encountering CAD remotely after TAVR will increase. The need for post-TAVR coronary angiography (CA) or percutaneous coronary intervention
(PCI) was thus far relatively rare amongst initial TAVR cohorts who were deemed either inoperable or at high-to-extreme surgical risk. These individuals often succumbed in the post-TAVR period to their burdensome comorbidities [3]. The present era, however, represents a period in TAVRs' evolution whereby the incidence for the need to perform CA and possible PCI after TAVR has considerably increased, posing new technical challenges for the coronary interventionalist.

Transcatheter heart valves (THV) can be broadly divided into balloon-, self- or mechanically expanding in nature [4], with each prosthetic class possessing unique characteristics 
pertaining to their design, material, annuloaortic orientation, and anatomic relation to the native coronary tree. These THV design differences may thus impact on how coronary interventionalists access the native coronary vasculature. Despite various reports describing technical differences and potential challenges of CA and PCI in TAVR recipients [5], the nuances and technicalities for catheterizing and effectively accessing the native epicardial coronary vasculature in the presence of in situ THV are yet to be systematically reported in the presence of a balloon-expandable valve system. The present analysis was thus aimed at reporting the feasibility and potential challenges faced when performing coronary procedures after TAVR with either the balloonexpandable Edwards-SAPIEN or the SAPIEN XT THVs.

\section{Materials and Methods}

2.1. Study Population. Figure 1 illustrates a detailed flowchart describing the selection process implemented to obtain the intended cohort of patients for this study. Over 1000 TAVRs were performed in the last decade at the Quebec Heart and Lung Institute, of which 616 patients had either an Edwards-SAPIEN or a SAPIEN XT THV implanted. From these patients, a total of 68 coronary procedures, either a CA or PCI, were achieved after TAVR. From a total of 30 postTAVR CAs performed, 2 CAs had insufficient data for complete analysis and were thus excluded, leaving a total of 28 patients available for analysis. Twenty-five patients underwent a PCI post-TAVR, but only 13 of these also had a pre-TAVR PCI with sufficient data to permitting complete analysis. All patients who presented with a combination of either pre- and post-TAVR CA $(n=28)$ or pre- and postTAVR PCI $(n=13)$ ultimately comprised the present study's population.

\subsection{Coronary Angiograms and Percutaneous Coronary} Interventions. Coronary angiograms were mandatory for all patients before TAVR. However, post-TAVR angiograms were requested by either the attending cardiologist responsible for each patient's clinical follow-up or by an emergency department cardiologist where patients were admitted. Percutaneous coronary interventions were executed or deferred prior to TAVR as decided by either the patients' primary cardiologist, the Heart Team (comprising of at least 1 interventional cardiologist and acardiac surgeon), or by an interventional cardiologist performing the $\mathrm{CA}$ in the setting of an acute coronary syndrome (ACS) before TAVR. The need for post-TAVR PCI was evaluated in a similar fashion to post-TAVR CA. Neither the CA nor the PCI performed before or after TAVR had to be necessarily performed by the same interventional cardiologist; however, all analyzed procedures were performed by an experienced interventional cardiologist from the same tertiary highvolume institution in which the TAVR procedures occurred. In fact, the interventional cardiologists responsible for the procedures assessed in the present study have had several years of experience as interventionalists, performing about 400 coronary angiograms and 250 percutaneous coronary

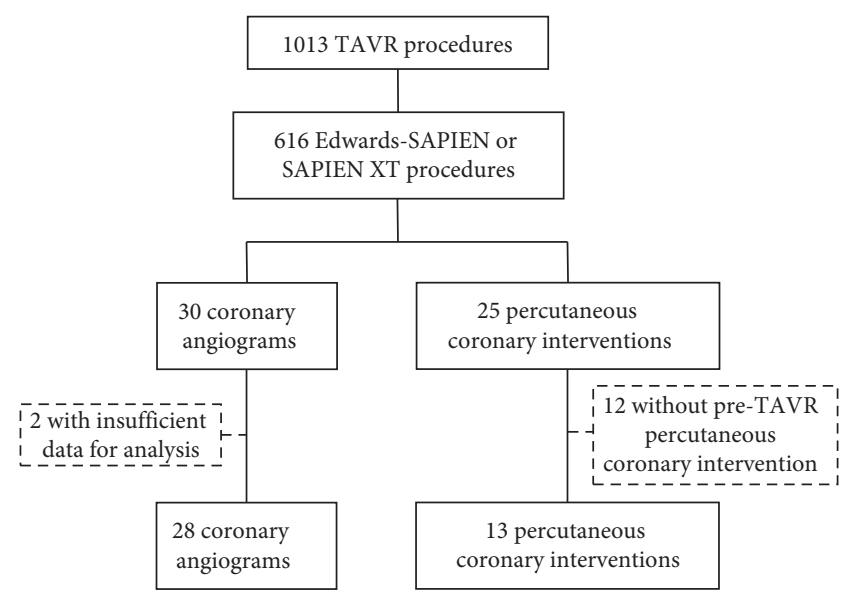

FIGURE 1: Flowchart of the study population.

interventions per interventionalist/year. All pre- and postTAVR procedures were reviewed by two experienced interventional cardiologists, and all relevant procedural and clinical characteristics were retrospectively gathered in a designated database for detailed analyses. The present study had no intention of comparing angiography to PCI, but rather to compare the same class of procedure performed in each patient before and after TAVR, since the comparison of technical aspects from two differing procedures might result in misleading findings.

2.3. Statistical Analysis. Two different sets of analyses were implemented in the present study, since 2 distinct groups were designed, one being the angiography group, in which only patients who had isolated CAs before and after TAVR were included, and the other being the PCI group, comprising patients who had PCI before and after TAVR. Categorical variables were assessed through the McNemar test and reported as absolute frequencies and percentages while continuous variables were analyzed using the Wilcoxon signed-rank sum test and are presented as mean \pm SD. Results were deemed significant when a 2-sided $p$-value below 0.05 was achieved. The Statistical Package for Social Sciences version 24 (SPSS Inc., IBM, Armonk, New York) was used to conduct all the statistical analysis.

\section{Results}

Table 1 describes baseline clinical characteristics and the TAVR procedural data for both the angiography and the PCI group. The presence of CAD was defined as prior coronary revascularization (percutaneous or surgical), prior myocardial infarction, or known coronary stenosis of at least $50 \%$ in diameter by visual evaluation. The THV position with respect to the coronary ostia is shown in Figure 2.

3.1. Coronary Angiograms. Post-TAVR CAs were performed at a mean time of $748 \pm 686$ days. The analyses of all aspects from the performed coronary angiograms, as seen in Table 2, showed that post-TAVR CA was more frequently performed 
TABLE 1: Baseline clinical characteristics and TAVR procedural data from the coronary angiogram group and the PCI group.

\begin{tabular}{|c|c|c|}
\hline & Angiogram group $(n=28)$ & PCI group $(n=13)$ \\
\hline \multicolumn{3}{|l|}{ Clinical characteristics } \\
\hline Age (years) & $72.54 \pm 9.08$ & $70.31 \pm 7.99$ \\
\hline Male & $14(50.0)$ & $9(69.2)$ \\
\hline Body mass index $\left(\mathrm{kg} / \mathrm{m}^{2}\right)$ & $28.94 \pm 5.96$ & $28.63 \pm 5.38$ \\
\hline NYHA functional class III-IV & $17(60.7)$ & $6(46.2)$ \\
\hline Diabetes & $13(46.4)$ & $5(38.5)$ \\
\hline Hypertension & $24(85.7)$ & $13(100)$ \\
\hline Coronary artery disease & $23(82.1)$ & $13(100)$ \\
\hline Prior percutaneous coronary intervention & $15(53.6)$ & $13(100)$ \\
\hline Prior coronary arterial bypass graft & $14(50.0)$ & $9(69.2)$ \\
\hline History of atrial fibrillation & $4(14.3)$ & $3(23.1)$ \\
\hline Peripheral vascular disease & $9(32.1)$ & $7(53.8)$ \\
\hline Chronic obstructive pulmonary disease & $7(25.0)$ & $5(38.5)$ \\
\hline Chronic kidney disease & $15(53.6)$ & $10(76.9)$ \\
\hline eGFR $(\mathrm{mL} / \mathrm{min})$ & $60.36 \pm 24.63$ & $58.47 \pm 26.01$ \\
\hline STS-PROM (\%) & $5.25 \pm 2.71$ & $5.89 \pm 3.06$ \\
\hline LogEuroSCORE (\%) & $17.22 \pm 11.79$ & $17.45 \pm 12.72$ \\
\hline Left coronary height (mm) & $12.77 \pm 3.27$ & $12.32 \pm 2.72$ \\
\hline Right coronary height (mm) & $13.23 \pm 3.20$ & $14.50 \pm 4.59$ \\
\hline Aortoventricular angulation (degrees) & $42.40 \pm 7.97$ & $43.30 \pm 7.50$ \\
\hline \multicolumn{3}{|l|}{ Procedural characteristics } \\
\hline Success & $28(100)$ & $13(100)$ \\
\hline \multicolumn{3}{|l|}{ Approach } \\
\hline Transfemoral & $8(28.6)$ & $4(30.8)$ \\
\hline Nontransfemoral & $20(71.4)$ & $9(69.2)$ \\
\hline \multicolumn{3}{|l|}{ Prothesis type } \\
\hline Edwards-SAPIEN & $12(42.9)$ & $6(46.2)$ \\
\hline SAPIEN XT & $16(57.1)$ & $7(53.8)$ \\
\hline \multicolumn{3}{|l|}{ Prothesis size (mm) } \\
\hline 23 & $15(53.6)$ & $5(38.5)$ \\
\hline 26 & $11(39.3)$ & $6(46.2)$ \\
\hline 29 & $2(7.1)$ & $2(15.4)$ \\
\hline \multicolumn{3}{|l|}{ THV-coronary ostia position } \\
\hline Supra-ostial & $5(17.8)$ & $3(23.0)$ \\
\hline Ostial level & $8(28.6)$ & $5(38.5)$ \\
\hline Infra-ostial & $15(53.6)$ & $5(38.5)$ \\
\hline
\end{tabular}

Variables are expressed as $n(\%)$ or mean \pm SD. eGFR $=$ estimated glomerular filtration rate; LogEuroSCORE $=$ logistic EuroSCORE predicted risk of mortality; NYHA = New York Heart Association; STS-PROM = Society of Thoracic Surgeons Predicted Risk of Mortality; THV = transcatheter heart valve.

in ACS scenarios, mainly due to unstable angina. The vast majority of procedures were performed through a radial approach (67.9\% pre-TAVR, $64.3 \%$ post-TAVR, $p=0.602)$.

Prior to TAVR, a mean of $1.21 \pm 0.69$ catheters/per patient was needed to cannulate the left coronary artery (LCA) which was most commonly (89\% of cases) achieved with a Judkins left (JL) catheter (Cordis Corporation, Freemont, CA, USA). In cases where cannulation was unachievable or deemed inappropriate, other catheters such as the Multipurpose (MP) or the Amplatz Left (AL) diagnostic catheters (Cordis Corporation, Freemont, CA, USA) were used as substitutes to the JL. A mean of $1.21 \pm 0.79$ catheters/per patient was used to perform the right coronary artery (RCA) angiogram before TAVR, and in $82 \%$ of cases, the Judkins Right (JR) catheter was used, while the Amplatz Right (AR) or the Barbeau diagnostic catheter were the most commonly used surrogates in case of failure with the first catheter. Noticeably, the implanted THV did not result in any increase in the number of catheters needed to perform CA after TAVR $(1.04 \pm 0.33$ catheters for the LCA and $0.96 \pm 0.58$ catheters for the RCA). Furthermore, the preferred choices of diagnostic catheters did not change significantly post-TAVR, with the JL and the JR diagnostic catheters being used in as much as $85 \%$ of the LCA angiograms and 75\% of RCA angiograms, respectively.

The achievement of selective contrast injection into the coronary arteries, the most frequent arterial access approach (radial), and the most used catheter diameter (5 French) chosen by the performing interventional cardiologists did not vary significantly after TAVR. Nevertheless, both the dose area product and the volume of contrast used were remarkably higher in the pre-TAVR procedures, as a larger number of coronary angiogram views and either a ventriculography and/or an aortogram were more likely performed in such procedures. After TAVR, in only one case out of 28, no LCA injection was attempted while the same happened in 5 cases involving the RCA, due to known chronic occlusion of these vessels, resulting in a mean of $0.96 \pm 0.58$ diagnostic catheters used for RCA angiography after TAVR (Table 2). 


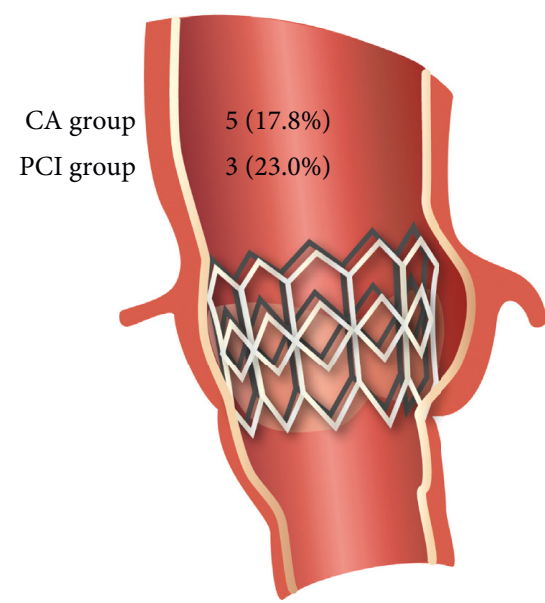

(a)

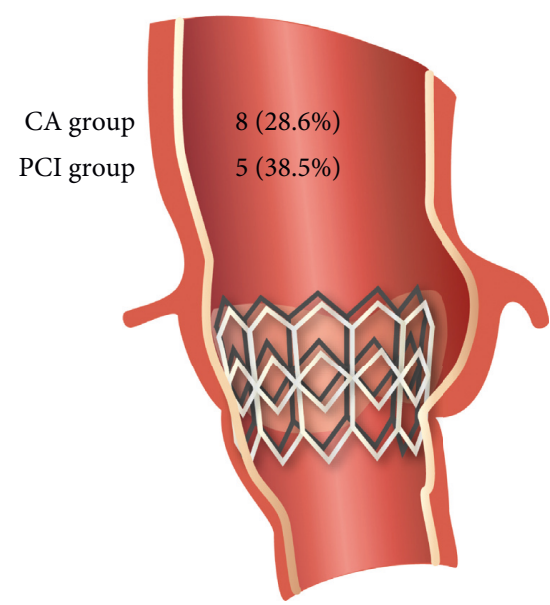

(b)

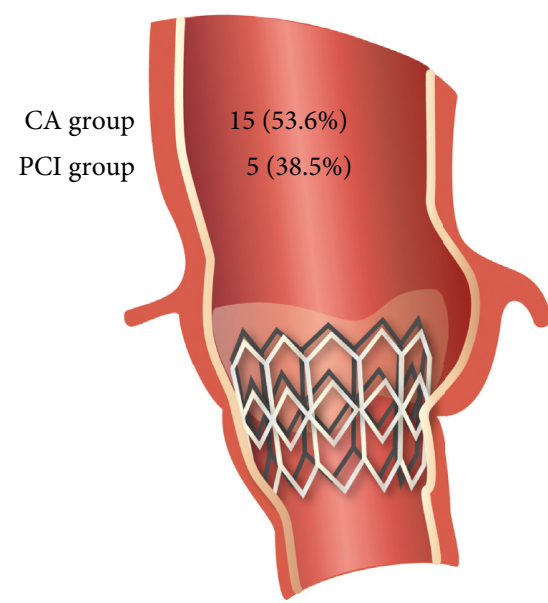

(c)

FIGURE 2: Diagram of the balloon-expandable transcatheter heart valve (THV) in regard to the coronary ostia. (a) Supra-ostial position of the THV (superior border of the stent frame above the lowest coronary artery ostia). (b) THV at the ostial level (superior border of the stent frame at the same level as the lowest coronary artery ostia). (c) Infra-ostial position of the THV (superior border of the stent frame below the lowest coronary artery ostia).

\subsection{Percutaneous Coronary Interventions. Post-TAVR PCIs} were performed at a mean time of $603 \pm 516$ days. Table 3 summarizes procedural indications, timing, and vessel/lesion characteristics. While pre-TAVR PCIs were performed mostly in stable circumstances, such as in asymptomatic/ silent ischemia or stable angina patients, PCIs after TAVR were mainly performed in ACS, such as non-ST elevation myocardial infarction (NSTEMI) or unstable angina, a pattern similar to that observed for the CA analysis.

All 11 cases of pre-TAVR left-sided PCI were performed with a 3.0 or 3.5 extra backup guiding catheter (XB) although a single change of curve size was observed in two of these cases. Post-TAVR left-sided PCI also had a XB guiding catheter chosen for 7 of 9 cases. In three of these cases, a change of catheter curve from 3.5 to 3.0 was observed, and two of them ended up being managed with a JL 3.5 guiding catheter. Pre-TAVR RCA PCI was achieved in only 2 cases with either a JR or an AL guiding catheter. Following TAVR, 3 other RCA PCIs were performed, again, with the same type of catheters or a Barbeau guiding catheter. Lack of the need to change the initially chosen guiding catheter was observed in all post-TAVR RCA PCIs. Of note, 2 patients had both pre- and post-TAVR vein graft PCI in which either an AL, an AR, or an MP guiding catheter was used. Despite the changes observed in both catheter sizes and types, a significant increase in the total number of guides needed to perform PCI was not observed (pre-TAVR: $1.15 \pm 0.38$; $\quad$ post-TAVR: $1.54 \pm 0.66$; $p=0.132)$.

Similar to the post-TAVR CA, no significant differences in achieving selective coronary injection or arterial access site preference (radial artery) were observed in post-TAVR PCI. Most guiding catheters had a $6 \mathrm{~F}$ diameter before and after TAVR, with type B2 or C lesions present in the left coronary bed being the most common lesion type treated during the vast majority of PCI in both the pre- and postTAVR periods. Other technical procedural aspects, namely, total procedural duration, fluoroscopy time, amount of contrast used and the dose area product also did not change after TAVR.

Although the type of stent, mainly drug-eluting stents, and number of lesions treated did not significantly differ between procedures, there was a significantly greater number of stents implanted pre-TAVR, which lead to a larger total stent length used in such procedures. Unlike what was observed during pre-TAVR PCI, no adjunctive instruments, such as the GuideLiner catheter (Vascular Solutions Inc., Minneapolis, MN, USA) or the rotational atherectomy system (Rotablator ${ }^{\mathrm{TM}}$, Boston Scientific, Marlborough, MA, USA), were used in the interventions after TAVR. Fluoroscopic/angiographic images of PCI before and after TAVR are shown in Figure 3.

\section{Discussion}

The present study demonstrates that CA and PCI performed following TAVR with a balloon-expandable Edwards valve did not exhibit significant changes in technical procedural aspects such as choice of arterial access site, catheter diameter, number of diagnostic or guiding catheters needed to perform the procedure, procedural duration, fluoroscopy time, contrast volume, and achievement of selective coronary injection compared with the respective pre-TAVR setting.

Aortic stenosis and coronary artery disease often coexist $[1,5-7]$, and updated international guidelines on coronary revascularization currently deem implementation of percutaneous coronary interventions on patients with symptomatic CAD prior to TAVR as appropriate $[8,9]$. This practice has in fact long been adopted not only in our institution but also in most centers worldwide [7, 10-14]. The rationale for providing complete revascularization for impending TAVR candidates is based on the notion of lowering the risk from coronary ischemia that may transiently occur during the TAVR procedure per se (i.e., during 
TABLE 2: Clinical and procedural characteristics for the coronary angiogram group $(n=28)$.

\begin{tabular}{|c|c|c|c|}
\hline & Pre-TAVR & Post-TAVR & $p$ value \\
\hline \multicolumn{4}{|l|}{ Clinical characteristics } \\
\hline Time from TAVR (days) & $428.57 \pm 1287.40$ & $748.75 \pm 686.62$ & - \\
\hline \multicolumn{4}{|l|}{ Procedure indication } \\
\hline Asymptomatic/silent ischemia & $21(75.0)$ & $10(35.7)$ & 0.008 \\
\hline Stable angina & $2(7.1)$ & $0(0)$ & - \\
\hline Unstable angina & $3(10.7)$ & $11(39.3)$ & 0.021 \\
\hline Non-ST elevation myocardial infarction & $2(7.1)$ & $5(17.9)$ & 0.257 \\
\hline ST elevation myocardial infarction & $0(0)$ & $2(7.1)$ & - \\
\hline \multicolumn{4}{|l|}{ Procedural characteristics } \\
\hline \multicolumn{4}{|l|}{ Access site } \\
\hline Femoral & $9(32.1)$ & $10(35.7)$ & 0.655 \\
\hline Radial & $19(67.9)$ & $18(64.3)$ & 0.602 \\
\hline \multicolumn{4}{|l|}{ Catheter diameter (French) } \\
\hline 4 & $0(0)$ & $2(7.1)$ & - \\
\hline 5 & $28(100)$ & $25(89.3)$ & - \\
\hline 6 & $0(0)$ & $1(3.6)$ & - \\
\hline \multicolumn{4}{|l|}{ Number of diagnostic catheters used } \\
\hline Left coronary artery & $1.21 \pm 0.69$ & $1.04 \pm 0.33$ & 0.219 \\
\hline Right coronary artery & $1.21 \pm 0.79$ & $0.96 \pm 0.58$ & 0.305 \\
\hline VG/LIMA & $0.79 \pm 0.96$ & $0.82 \pm 0.94$ & 1.000 \\
\hline \multicolumn{4}{|l|}{ Number of guidings needed } \\
\hline Left coronary artery & $0.14 \pm 0.36$ & $0 \pm 0$ & 0.125 \\
\hline Right coronary artery & $0 \pm 0$ & $0 \pm 0$ & - \\
\hline \multicolumn{4}{|l|}{ Selective injection achieved } \\
\hline Left coronary artery & $25(92.6)$ & $22(81.5)$ & 0.257 \\
\hline Right coronary artery & $22(95.7)$ & $19(82.6)$ & 0.083 \\
\hline Number of angiographic views & $12.27 \pm 4.71$ & $9.85 \pm 3.03$ & 0.004 \\
\hline Ventriculography \pm aortogram performed & $22(78.6)$ & $11(39.3)$ & 0.008 \\
\hline Procedural duration (min) & $24.86 \pm 10.85$ & $23.43 \pm 8.36$ & 0.877 \\
\hline Fluoroscopy time (min) & $7.59 \pm 6.06$ & $6.23 \pm 4.20$ & 0.269 \\
\hline Dose area product $\left(\mathrm{cGy} / \mathrm{cm}^{2}\right)$ & $6295.88 \pm 5125.99$ & $4252.11 \pm 2255.88$ & 0.008 \\
\hline Contrast amount $(\mathrm{mL})$ & $125.32 \pm 58.27$ & $83.79 \pm 40.77$ & 0.003 \\
\hline
\end{tabular}

Variables are expressed as $n(\%)$ or mean \pm SD. LIMA = left internal mammary artery; VG= venous graft.

rapid ventricular pacing and aortic balloon inflation) $[7,10,12]$ along with improving symptoms and clinical outcomes post-TAVR.

However, a recent meta-analysis has suggested that revascularization pre-TAVR does not seem to award any clinical advantage and might actually be associated with an increased risk of major vascular complications and 30-day mortality [12]. More importantly, however, appears to be the acknowledgement that TAVR has been progressively implemented in lower surgical risk patients [15-21], who are frequently younger and might not display clinically relevant CAD yet, but who may require future CA or PCI [14]. Such observations have raised concerns regarding the feasibility and possible challenges encountered when performing postTAVR CAs and PCIs and their relation to the different types of THVs.

It is estimated that 3 to $7 \%$ of TAVR recipients undergo CA or PCI at midterm follow-up post-TAVR [7, 10, 14, 22, 23]. Diverse indications for post-TAVR coronary procedures have been portrayed in literature and in a few cohorts, such as the one presented, acute coronary syndromes and development or progression of angina pectoris were the leading causes for such procedures $[7,10,14,24]$. This observation is key for interpreting possible differences amongst coronary procedures performed before and after TAVR, as procedures performed in ACS patients aim to identify and treat culprit lesions only, while pre-TAVR procedures are frequently performed in stable patients potentially targeting a more complete revascularization. This may partially explain the higher number of stents and total stent length in the pre-TAVR PCI procedures. Interestingly, most PCI procedures after TAVR were secondary to an ACS, mainly non-ST elevation myocardial infarction.

Fundamental differences exist amongst the several types of commercially available THVs, and a detailed knowledge of such characteristics and the potential challenges they impose on future coronary interventions is paramount $[7,10,14]$. Valves with a stent frame extending beyond the coronary ostia, as the self-expanding CoreValve series (Medtronic, Minneapolis, Minnesota, USA), have their structure designed with a central concavity as well as open cells that permit selective coronary catheterization. Balloon-expandable valves, such as the SAPIEN valve series (Edwards Lifesciences, Irvine, California, USA), are most frequently positioned in a subcoronary position or just at the level of the coronary ostia, as was observed in our study, and as such are expected to have less of an impact on coronary catheterization $[5,7,10,23]$. The aortoventricular angulation, obtained by measuring the angle between the horizontal plane and the aortic annulus [25], plays an 
Table 3: Clinical, lesion, and procedural characteristics for the PCI group $(n=13)$.

\begin{tabular}{|c|c|c|c|}
\hline & Pre-TAVR & Post-TAVR & $p$ value \\
\hline \multicolumn{4}{|l|}{ Clinical characteristics } \\
\hline Time from TAVR (days) & $37.31 \pm 39.38$ & $603.92 \pm 516.58$ & - \\
\hline \multicolumn{4}{|l|}{ Procedure indication } \\
\hline Asymptomatic/silent ischemia & $9(69.2)$ & $2(15.4)$ & 0.008 \\
\hline Stable angina & $2(15.4)$ & $1(7.7)$ & 0.564 \\
\hline Unstable angina & $2(15.4)$ & $4(30.8)$ & 0.414 \\
\hline Non-ST elevation myocardial infarction & $0(0)$ & $6(46.2)$ & - \\
\hline ST elevation myocardial infarction & $0(0)$ & $0(0)$ & - \\
\hline \multicolumn{4}{|l|}{ Procedural characteristics } \\
\hline \multicolumn{4}{|l|}{ Vessel treated } \\
\hline Left main & $6(46.2)$ & $2(15.4)$ & 0.046 \\
\hline Left anterior descending artery & $5(38.5)$ & $4(30.8)$ & 0.564 \\
\hline Circumflex artery & $7(53.9)$ & $4(30.8)$ & 0.083 \\
\hline Ramus intermedius & $2(15.4)$ & $2(15.4)$ & 1 \\
\hline Right coronary artery & $2(15.4)$ & $3(23.1)$ & 0.317 \\
\hline Venous graft & $2(15.4)$ & $2(15.4)$ & 1.000 \\
\hline \multicolumn{4}{|l|}{ Lesion location } \\
\hline Ostial & $7(53.9)$ & $6(46.2)$ & 0.564 \\
\hline Bifurcation & $7(53.9)$ & $4(30.8)$ & 0.180 \\
\hline Type B2/C lesion & $12(92.3)$ & $11(84.6)$ & 0.564 \\
\hline Diameter stenosis (\%) & $83.17 \pm 12.13$ & $89.31 \pm 11.88$ & 0.267 \\
\hline \multicolumn{4}{|l|}{ TIMI flow before PCI } \\
\hline 0 & $1(7.7)$ & $1(7.7)$ & 1 \\
\hline 1 & $0(0)$ & $1(7.7)$ & - \\
\hline 2 & $1(7.7)$ & $2(15.4)$ & 0.564 \\
\hline 3 & $11(84.6)$ & $9(69.2)$ & 0.414 \\
\hline \multicolumn{4}{|l|}{ TIMI flow after PCI } \\
\hline 0 & $0(0)$ & $0(0)$ & - \\
\hline 1 & $0(0)$ & $1(7.7)$ & - \\
\hline 2 & $0(0)$ & $0(0)$ & - \\
\hline 3 & $13(100)$ & $12(92.3)$ & - \\
\hline \multicolumn{4}{|l|}{ Access site } \\
\hline Femoral & $3(23.1)$ & $4(30.8)$ & 0.655 \\
\hline Radial & $10(76.9)$ & $8(61.5)$ & 0.414 \\
\hline Both & $0(0)$ & $1(7.7)$ & - \\
\hline \multicolumn{4}{|l|}{ Catheter diameter (French) } \\
\hline 5 & $3(23.1)$ & $6(46.2)$ & 0.257 \\
\hline 6 & $8(61.5)$ & $7(53.89)$ & 0.655 \\
\hline 7 & $2(15.4)$ & $0(0)$ & - \\
\hline Number of guidings needed & $1.15 \pm 0.38$ & $1.54 \pm 0.66$ & 0.132 \\
\hline Selective injection achieved & $12(92.3)$ & $13(100)$ & - \\
\hline Number of lesions treated & $1.85 \pm 0.90$ & $1.31 \pm 0.48$ & 0.180 \\
\hline Number of stents implanted & $2.31 \pm 1.55$ & $1.31 \pm 0.75$ & 0.037 \\
\hline Total stent length $(\mathrm{mm})$ & $57.23 \pm 46.65$ & $25.69 \pm 18.06$ & 0.018 \\
\hline Maximum stent diameter $(\mathrm{mm})$ & $3.15 \pm 0.47$ & $3.13 \pm 1.07$ & 0.680 \\
\hline \multicolumn{4}{|l|}{ Type of stent used } \\
\hline Bare-metal stent & $3(23.1)$ & $1(7.7)$ & 0.317 \\
\hline Drug-eluting stent & $10(76.9)$ & $11(84.6)$ & 0.655 \\
\hline Balloon predilation & $13(100)$ & $11(84.6)$ & - \\
\hline Balloon postdilation & $11(84.6)$ & $9(69.2)$ & 0.414 \\
\hline GuideLiner used & $1(7.7)$ & $0(0)$ & - \\
\hline Rotational atherectomy performed & $4(30.8)$ & $0(0)$ & - \\
\hline Aspiration thrombectomy & $0(0)$ & $1(7.7)$ & - \\
\hline Number of angiographic views & $16.46 \pm 7.97$ & $15.07 \pm 6.00$ & 0.667 \\
\hline Fluoroscopy time (min) & $20.99 \pm 20.92$ & $20.69 \pm 11.94$ & 0.677 \\
\hline Procedural duration (min) & $63.15 \pm 37.41$ & $65.23 \pm 26.38$ & 0.672 \\
\hline Dose area product $\left(\mathrm{cGy} / \mathrm{cm}^{2}\right)$ & $13166 \pm 8056$ & $8098 \pm 5579$ & 0.131 \\
\hline Contrast amount $(\mathrm{mL})$ & $170 \pm 86.98$ & $148.92 \pm 58.85$ & 0.748 \\
\hline Procedural success & $13(100)$ & $12(92.3)$ & - \\
\hline
\end{tabular}

Variables are expressed as $n(\%)$ or mean $\pm \mathrm{SD}$. $\mathrm{PCI}=$ percutaneous coronary intervention. 


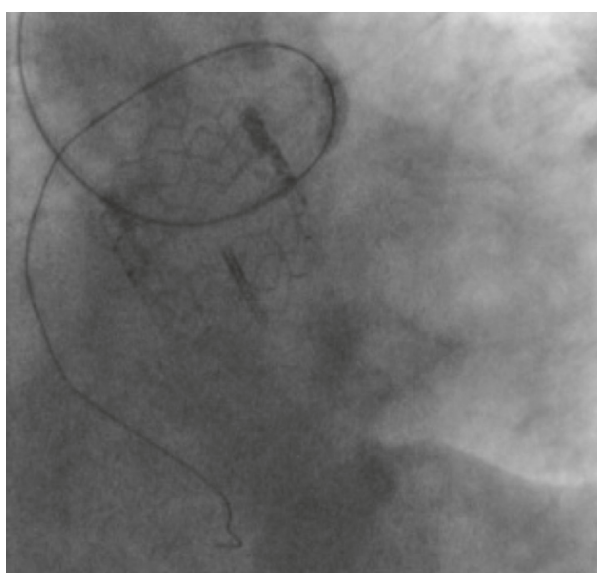

(a)

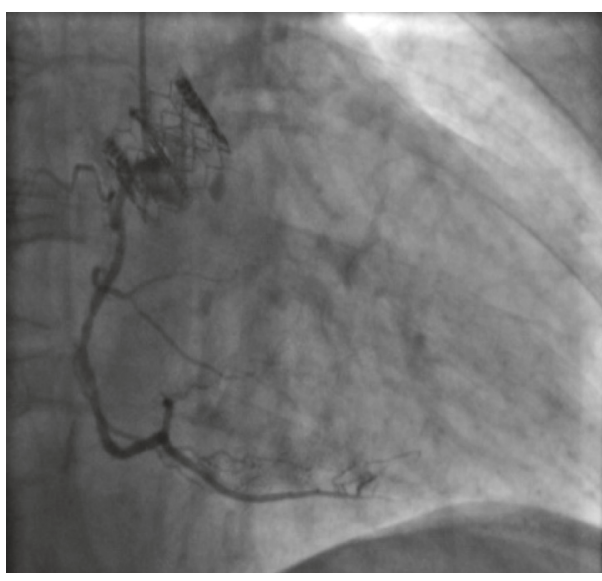

(c)

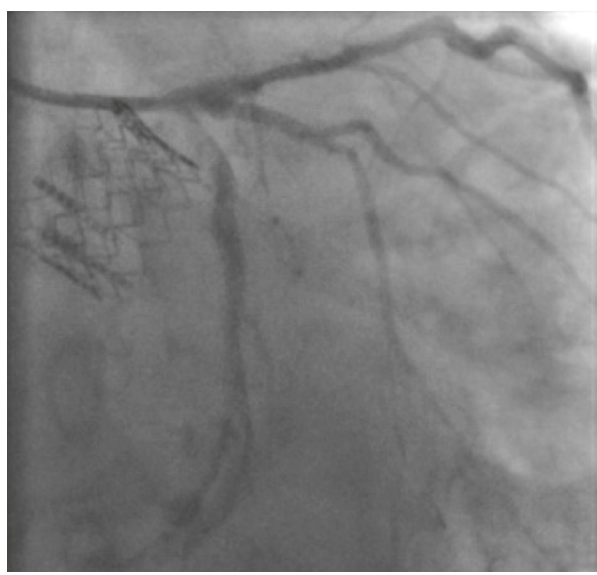

(b)

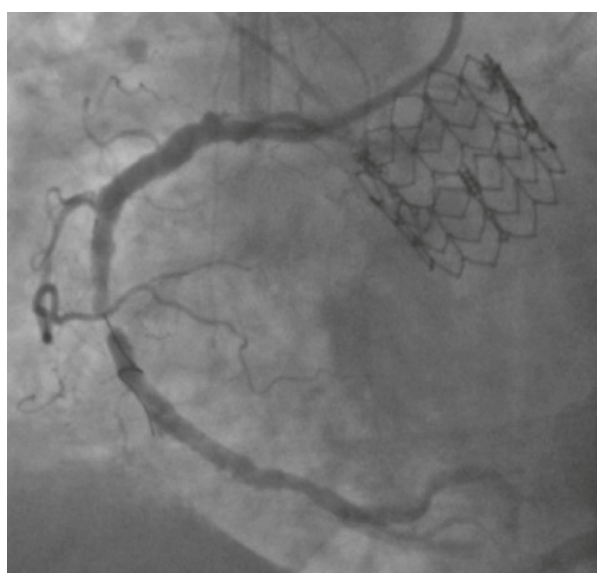

(d)

Figure 3: Post-TAVR PCI cases. (a) 5-French extra backup guiding catheter used during a PCI performed through the THV struts. (b) 6-French extra backup guiding catheter used during a PCI performed over the THV struts. (c) 5-French Judkins right guiding catheter used during a PCI performed through the THV struts. (d) 6-French Barbeau guiding catheter used during a PCI performed over the THV struts.

important role in both the delivery of the THV and its relation to the coronary ostia, particularly in cases where a long THV is implanted [26]. Large angulations found in horizontal aortas might prevent the achievement of a perpendicular deployment of THVs with long stent frames, potentially leading to a more extensive interaction with the coronary ostia. The balloon-expandable valves used in our study, however, present a much shorter stent frame height $(\sim 14$ to $19 \mathrm{~mm})$ and thus, are less likely to have a significant interaction with the coronary arteries due to the aortoventricular angulation. Moreover, as presented in Table 1, both the angiogram and PCI groups presented a mean aortoventricular angulation below the usual threshold determining a problematic THV positioning [25].

An increase in the number of diagnostic or guiding catheters, procedural duration, fluoroscopy time, or different choice of arterial access was not found during CA or PCI in our cohort of balloon-expandable valve recipients, as has been traditionally described in the context of self-expanding valve coronary procedures $[7,10,14,22]$. Interestingly, the dose area product and contrast amount were actually smaller during post-TAVR CA performed in our cohort, contrary to what has been found in self-expanding valve cohorts $[10,14]$. Probable reasons for such findings are that, as expected, ventriculography and/or aortogram were significantly less likely to be executed after TAVR and a lower number of angiographic views were performed after TAVR. Nonetheless, a trend of less-frequent achievement of coronary selective injections, similar to what is observed in some CoreValve series $[7,14]$, was present in this cohort.

Post-TAVR PCIs in patients with self-expanding valves have been primarily undertaken through the femoral access approach $[10,22]$; still, the radial access approach to PCI has been the preferred route at our institution for over 2 decades. The present analysis demonstrates that such a predilection for the transradial access remained unchanged despite the presence of a prior balloon-expandable THV. Six-French systems remained the most commonly used guiding catheters diameters after TAVR $[14,22]$ although 5 -French guiding catheters were numerically twice as frequent during post-TAVR PCI in the present study. Interestingly, the treated coronary lesions after TAVR were mainly situated within the left coronary bed both in our cohort and in previously reported studies [10, 14, 22, 23]. A 
significantly smaller number of stents, as well as total stent length during post-TAVR in our studied population, were observed. This observation might be explained by the commonly observed practice of performing PCI in all lesions deemed significant prior to the TAVR procedure $[7,10-14]$.

As previously mentioned, inherent THV characteristics must be acknowledged to better understand the possible challenges presented in post-TAVR coronary procedures. Yudi et al. [5] have recently proposed a useful algorithm for CA and PCI after TAVR. While JL and JR catheters are considered the primary choices for performing CA regardless of the THV type, particularities such as using the J-wire to enter the diamond in front of the coronary ostia and the possibility of using a JR4 to cannulate the LCA might be beneficial in CoreValve recipients. Failure to perform selective injections with these catheters should be followed by the use of guiding catheters, such as the $\mathrm{XB} /$ Voda Left/Femoral Left for SAPIEN recipients or the Ikari Right/Femoral Left/MP for CoreValve recipients, which allow for better support, particularly with the concomitant coronary wiring and the possibility to perform a balloonassisted tracking or to use a guide extension catheter regardless of the THV type. No specific guiding catheter was assigned as the first choice for PCI in a balloon-expandable THV recipient; however, the XB guiding catheter may bend and should be maneuvered carefully. In CoreValve recipients, the JL for the LCA, or the JR for the RCA, is the preferred choice. Interestingly, the majority of the aforementioned suggestions are in accordance with our findings, in which the JL/JR catheters were most commonly used for post-TAVR CA and rare changes in guiding catheter choices were observed after the implantation of a balloonexpandable THV. The technical similarities observed in our procedures, performed prior to the publication of suggestions, might be due to the high level of expertise of the interventional cardiologists involved in our study, who have performed numerous CAs and PCIs over several years of practice, most with over a decade of experience in a highvolume institution.

Several limitations of the current analyses warrant consideration. Although instruments and equipment were equally available at the time of the compared procedures, as previously stated, there was a limited number of procedures available for comparison and they were not necessarily performed by the same interventional cardiologist; as such, despite having been performed by experienced interventional cardiologists from a single high-volume tertiary institution, one may argue that the level of expertise may vary amongst the performing physicians. Furthermore, the difference in the clinical scenarios in which the procedures were performed, namely, the greater prevalence of ACS on post-TAVR procedures, could have influenced some of the results since in such scenarios, there is less time for planning and choosing the most appropriate material to undertake both the CA and PCI. The intention to achieve a complete revascularization prior to TAVR is seldomly observed in ACS presented by TAVR recipients, where the objective is essentially to diagnose and treat the culprit lesion. Additionally, one must not disregard the retrospective nature of the present study and its inherent limitations.

\section{Conclusion}

In conclusion, following TAVR with a balloon-expandable Edwards-SAPIEN or SAPIEN XT valve, both CA and PCI are feasible and may be undertaken without substantial interaction with the implanted THV.

\section{Data Availability}

The data used to support the findings of this study are available from the corresponding author upon request.

\section{Disclosure}

All authors listed meet the authorship criteria according to the latest guidelines of the International Committee of Medical Journal Editors and are in agreement with the manuscript.

\section{Conflicts of Interest}

Dr. Josep Rodés-Cabau has received research grants from Edwards Lifesciences, Medtronic, and Boston Scientific. The other authors do not report any potential conflicts of interest.

\section{References}

[1] L. Faroux, L. Guimaraes, J. Wintzer-Wehekind et al., "Coronary artery disease and transcatheter aortic valve replacement: JACC state-of-the-art review," Journal of the American College of Cardiology, vol. 74, no. 3, pp. 362-372, 2019.

[2] R. Puri, C. Chamandi, T. Rodriguez-Gabella, and J. RodésCabau, "Future of transcatheter aortic valve implantation-evolving clinical indications," Nature Reviews Cardiology, vol. 15, no. 1, pp. 57-65, 2018.

[3] R. Puri, B. Iung, D. J. Cohen, and J. Rodés-Cabau, “TAVI or no TAVI: identifying patients unlikely to benefit from transcatheter aortic valve implantation," European Heart Journal, vol. 37, no. 28, pp. 2217-2225, 2016.

[4] C. Chamandi, R. Puri, T. Rodriguez-Gabella, and J. RodésCabau, "Latest-generation transcatheter aortic valve replacement devices and procedures," Canadian Journal of Cardiology, vol. 33, no. 9, pp. 1082-1090, 2017.

[5] M. B. Yudi, S. K. Sharma, G. H. L. Tang, and A. Kini, "Coronary angiography and percutaneous coronary intervention after transcatheter aortic valve replacement," Journal of the American College of Cardiology, vol. 71, no. 12, pp. 1360-1378, 2018.

[6] J.-M. Paradis, J. Fried, T. Nazif et al., "Aortic stenosis and coronary artery disease: what do we know? What don't we know? A comprehensive review of the literature with proposed treatment algorithms," European Heart Journal, vol. 35 , no. 31, pp. 2069-2082, 2014.

[7] J. Blumenstein, W.-K. Kim, C. Liebetrau et al., "Challenges of coronary angiography and intervention in patients previously treated by TAVI," Clinical Research in Cardiology, vol. 104, no. 8, pp. 632-639, 2015. 
[8] P. Kolh, S. Windecker, F. Alfonso et al., "2014 ESC/EACTS guidelines on myocardial revascularization: the task force on myocardial revascularization of the European society of cardiology (ESC) and the European association for cardiothoracic surgery (EACTS). Developed with the special contribution of the European association of percutaneous cardiovascular interventions (EAPCI)," European Journal of Cardio-Thoracic Surgery, vol. 46, no. 4, pp. 517-592, 2014.

[9] M. R. Patel, J. H. Calhoon, G. J. Dehmer et al., "Acc/AATS/ AHA/ASE/ASNC/SCAI/SCCT/STS 2017 appropriate use criteria for coronary revascularization in patients with stable ischemic heart disease: a report of the American college of cardiology appropriate use criteria task force, American association for thoracic surgery, American heart association, American society of echocardiography, American society of nuclear cardiology, society for cardiovascular angiography and interventions, society of cardiovascular computed tomography, and society of thoracic surgeons," Journal of the American College of Cardiology, vol. 69, no. 17, pp. 2212-2241, 2017.

[10] W. W. Htun, C. Grines, and T. Schreiber, "Feasibility of coronary angiography and percutaneous coronary intervention after transcatheter aortic valve replacement using a medtronic self-expandable bioprosthetic valve," Catheterization and Cardiovascular Interventions, vol. 91, no. 7, pp. 1339-1344, 2017.

[11] Y. Abramowitz, S. Banai, G. Katz et al., "Comparison of early and late outcomes of TAVI alone compared to TAVI plus PCI in aortic stenosis patients with and without coronary artery disease," Catheterization and Cardiovascular Interventions, vol. 83, no. 4, pp. 649-654, 2014.

[12] R. A. Kotronias, C. S. Kwok, S. George et al., "Transcatheter aortic valve implantation with or without percutaneous coronary artery revascularization strategy: a systematic review and meta-analysis," Journal of the American Heart Association, vol. 6, no. 6, 2017.

[13] M. M. Mahmood and D. F. Muir, "Management strategies for severe aortic stenosis and coronary artery disease in the transcatheter aortic valve implantation era," Continuing Cardiology Education, vol. 3, no. 1, pp. 4-10, 2017.

[14] M. Boukantar, R. Gallet, G. Mouillet et al., "Coronary procedures after TAVI with the self-expanding aortic bioprosthesis medtronic corevalve ${ }^{\mathrm{TM}}$, not an easy matter," Journal of Interventional Cardiology, vol. 30, no. 1, pp. 56-62, 2017.

[15] D. H. Adams, J. J. Popma, M. J. Reardon et al., "Transcatheter aortic-valve replacement with a self-expanding prosthesis," New England Journal of Medicine, vol. 370, no. 19, pp. 1790-1798, 2014.

[16] M. B. Leon, C. R. Smith, M. Mack et al., "Transcatheter aorticvalve implantation for aortic stenosis in patients who cannot undergo surgery," New England Journal of Medicine, vol. 363, no. 17 , pp. 1597-1607, 2010.

[17] M. B. Leon, C. R. Smith, M. J. Mack et al., "Transcatheter or surgical aortic-valve replacement in intermediate-risk patients," The New England Journal of Medicine, vol. 374, no. 17, pp. 1609-1620, 2016.

[18] R. A. Nishimura, C. M. Otto, R. O. Bonow et al., "2017 AHA/ ACC focused update of the 2014 AHA/ACC guideline for the management of patients with valvular heart disease: a report of the American college of cardiology/American heart association task force on clinical practice guidelines," Circulation, vol. 135, no. 25, pp. e1159-e1195, 2017.

[19] M. J. Reardon, N. M. Van Mieghem, J. J. Popma et al., "Surgical or transcatheter aortic-valve replacement in intermediate-risk patients," New England Journal of Medicine, vol. 376, no. 14, pp. 1321-1331, 2017.

[20] C. R. Smith, M. B. Leon, M. J. Mack et al., "Transcatheter versus surgical aortic-valve replacement in high-risk patients," New England Journal of Medicine, vol. 364, no. 23, pp. 2187-2198, 2011.

[21] H. G. H. Thyregod, D. A. Steinbrüchel, N. Ihlemann et al., "Transcatheter versus surgical aortic valve replacement in patients with severe aortic valve stenosis: 1-year results from the all-comers NOTION randomized clinical trial," Journal of the American College of Cardiology, vol. 65, no. 20, pp. 2184-2194, 2015.

[22] A. Allali, M. El-Mawardy, B. Schwarz et al., "Incidence, feasibility and outcome of percutaneous coronary intervention after transcatheter aortic valve implantation with a self-expanding prosthesis. Results from a single center experience," Cardiovascular Revascularization Medicine, vol. 17, no. 6, pp. 391-398, 2016.

[23] S. Katsanos, P. Debonnaire, F. van der Kley et al., "Position of Edwards SAPIEN transcatheter valve in the aortic root in relation with the coronary ostia," Catheterization and Cardiovascular Interventions, vol. 85, no. 3, pp. 480-487, 2015.

[24] V. Vilalta, L. Asmarats, A. Ferreira-Neto et al., "Incidence, clinical characteristics, and impact of acute coronary syndrome following transcatheter aortic valve replacement," Journal of the American College of Cardiolgy: Cardiovascular Interventions, vol. 11, no. 24, pp. 2523-2533, 2018.

[25] Y. Abramowitz, Y. Maeno, T. Chakravarty et al., "Aortic angulation attenuates procedural success following self-expandable but not balloon-expandable TAVR," JACC: Cardiovascular Imaging, vol. 9, no. 8, pp. 964-972, 2016.

[26] D. E. Litmanovich, E. Ghersin, D. A. Burke, J. Popma, M. Shahrzad, and A. A. Bankier, "Imaging in transcatheter aortic valve replacement (TAVR): role of the radiologist," Insights into Imaging, vol. 5, no. 1, pp. 123-145, 2014. 


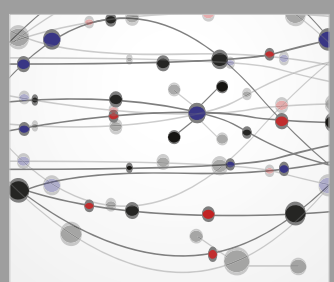

The Scientific World Journal
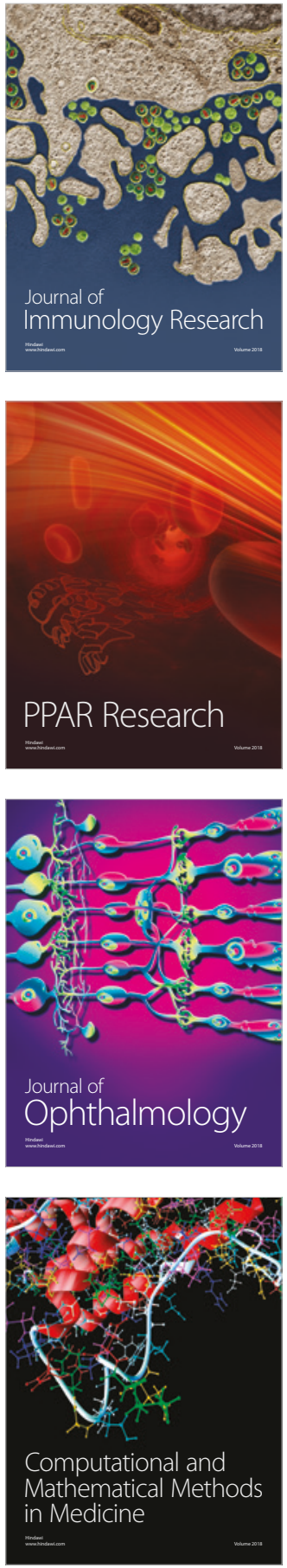

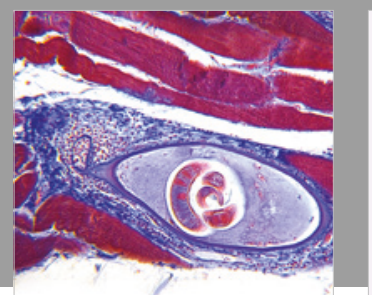

Gastroenterology Research and Practice

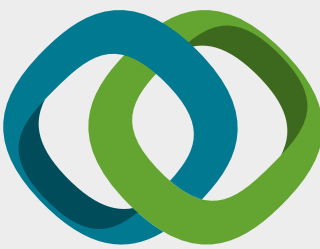

\section{Hindawi}

Submit your manuscripts at

www.hindawi.com
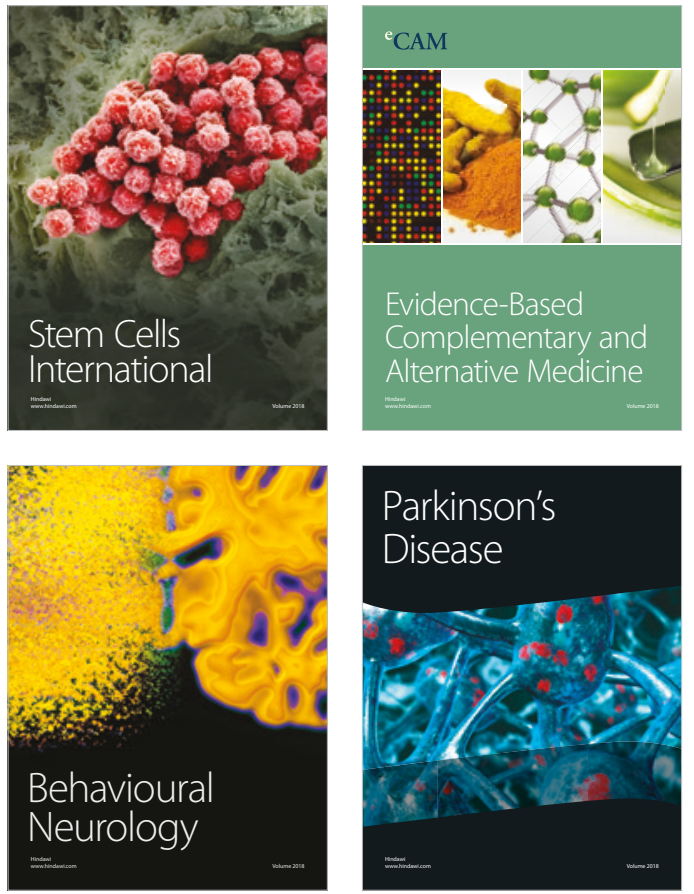

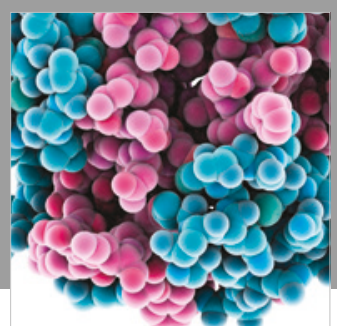

ournal of

Diabetes Research

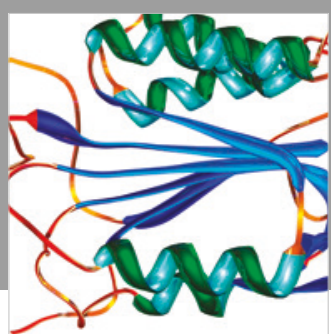

Disease Markers
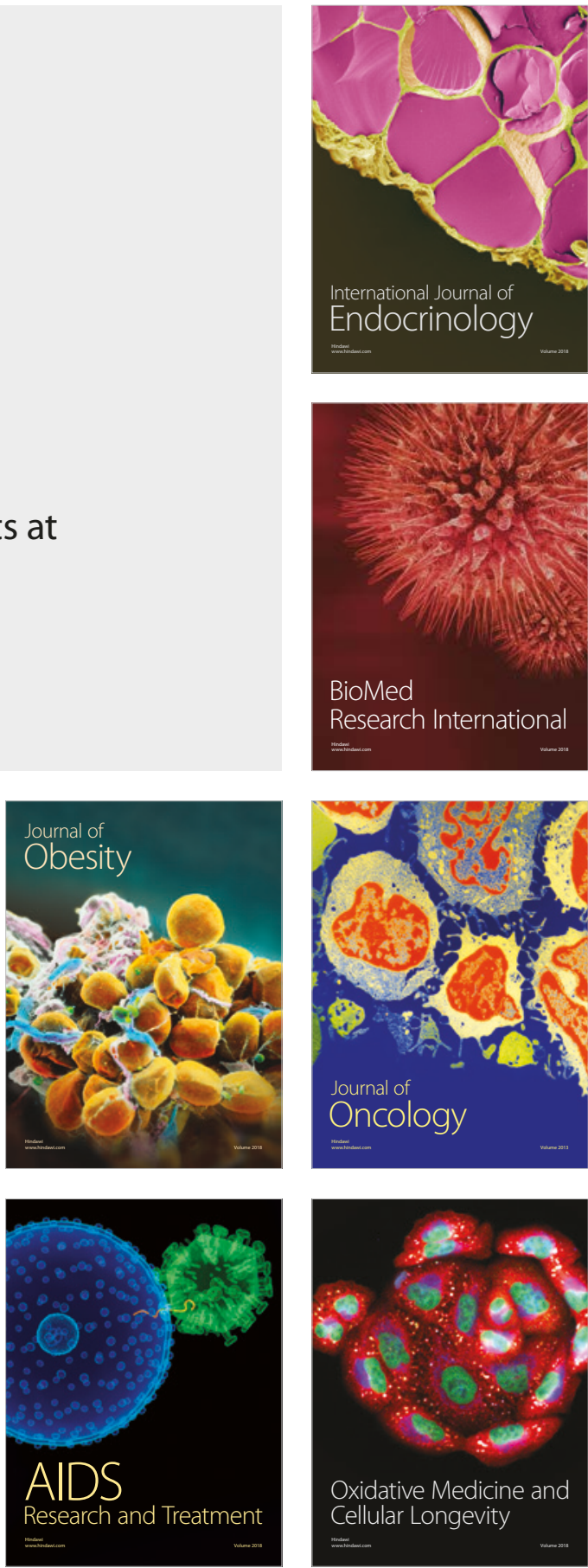